Check for updates

Cite this: RSC Adv., 2017, 7, 30673

Received 28th March 2017 Accepted 12th May 2017

DOI: $10.1039 / c 7 r a 03563 b$

rsc.li/rsc-advances

\section{Influence of magnetohydrodynamic viscous flow on entropy generation within porous micro duct using the Lattice Boltzmann Method}

\author{
Raja Rabhi, (D) * Abir Yahya, Bayssain Amami and Hacen Dhahri \\ In this work entropy generation and heat transfer for magnetohydrodynamic (MHD) forced convection flow \\ in a micro duct filled with a porous medium are investigated using a modified axisymmetric Lattice \\ Boltzmann Method. In modeling the flow, the Brinkman-Forchheimer-extended Darcy model is \\ incorporated in momentum equations. The Local Thermal Non-Equilibrium (LTNE) assumption between \\ fluid and solid phases is adopted to investigate heat transfer. All terms of viscous dissipation effects are \\ included in the energy equation of the fluid phase. Our attention is focused on the effects of different \\ values of Hartmann number $\mathrm{Ha}$, Knudsen number, Kn, Eckert number, Ec, and Biot number, Bi, on the \\ flow, Nusselt number and entropy generation.
}

\section{Introduction}

The flow phenomenon in a porous microfluidic channel has been analyzed by many researchers. This is thanks to its important applications in biochemistry, biomedicines, fuel cells and heat exchange. ${ }^{1,2}$ The fluid flow in these fields is effectively actuated by electrical fields, magnetic fields, or their suitable combinations. Among the different effects, magnetohydrodynamic (MHD) micro-systems have attracted the attention of many researchers $\mathrm{s}^{3,4}$ due to their prospective applications in microfluidic systems in recent years. Jian ${ }^{5}$ provided the transient MHD heat transfer and entropy generation in a micro-parallel channel combined with pressure and electro-osmotic effects. Das et al. ${ }^{6}$ illustrated certain applications of the MHD pumps such as sample injection, fluid flow in packs bed and on-chip assay development. Ellahi ${ }^{7}$ studied the magnetohydrodynamic flow of non-Newtonian nano fluids in a pipe and discussed the effects of various physical parameters on fluid flow and heat transfer. He demonstrates that the Hartmann number Ha, reduces the fluid motion and that the velocity profile is larger than that of the temperature profile. Aminossadati et al. ${ }^{8}$ investigated the effects of magnetic field on nanofluid forced convection in a partially heated micro channel. In their study, the Local thermal Equilibrium (LTE) model is used and the effect of the viscous dissipation is neglected.

Due to the complex structure of porous metals together with the computational consumption, the molecular dynamic method is limited to a volume containing finite particles. As

National School of Engineers, Laboratory of Thermal and Energy Systems Studies, Monastir University, Ibn Eljazzar Street, 5019 Monastir, Tunisia. E-mail: rajarabhi@gmail.com a novel numerical approach, the Lattice Boltzmann Method (LBM) is introduced to investigate the structures of the ferrofluid. ${ }^{9,10}$ Recently, the Lattice Boltzmann Method originating from the kinetic Boltzmann equation became popular over the past few years. It considers a fluid as a set of artificial particles and explores the microscopic features of the fluid by using the collision effect among these particles. There are two Lattice Boltzmann models for simulating MHD flows: the multispeed (MS) model and multi-distribution function (MDF) model. In the MDF model, presented by Dellar, ${ }^{11,12}$ the Lorentz force can be introduced as a point-wise force, the induction equation is also solved using an Lattice BhatnagarGross-Krook model (LBGK) equation by introducing an independent distribution function. MDF models can improve the numerical stability. ${ }^{13,14}$

The second law of thermodynamics explains that energy is moves from high quality energy to low quality energy. Quality of energy during a process also considers the quantitative measurement of exergy, potential work and entropy, and nonpotential work. Quality of energy during a process has also been described in the second law of thermodynamics. Entropy generation is responsible for system performance as this is proportional to the energy destruction. Most of entropy generation studies on porous media use the volume-averaged entropy generation which assumes the solid and fluid phases to be at thermal equilibrium. In the up-to-date literature, there are few studies on the entropy generation of porous media flow which consider the thermal non-equilibrium condition between the two phases in porous micro systems. ${ }^{15-20}$ Ting et al. ${ }^{15}$ studied the thermal non-equilibrium entropy generation based on differential method flow in a porous microchannel. A numerical study used a thermal non-equilibrium model to investigate the entropy generation 
of natural convection in a saturated porous cavity treated by Baytas. ${ }^{16}$ By using the same model, entropy generation analyses were performed on rarefied gaseous slip flows (Buonomo et $a l .{ }^{17}$ ) and partially-filled porous media flows (Torabi et al. ${ }^{18}$ ). Similarly to previous publications, Ting et al. ${ }^{19}$ treated entropy generation of viscous dissipative nanofluid convection in asymmetrically heated porous microchannels with solidphase heat generation. Moreover, Betchen and Straatman ${ }^{20}$ derived the volume-averaged form of entropy generation function for non-equilibrium heat transfer in high conductivity porous foams.

In sight of the limitations in the previous works, in this paper, a modified axisymmetric LBM scheme is employed to investigate the physics of flow, fluid-solid coupling heat transfer and entropy generation in a micro duct filled with porous medium including viscous dissipation effects under the local thermal non equilibrium assumptions. Furthermore, new developments concerns the magnetic field is used in order to investigate the magnetic field behavior.

The rest of the paper is organized as follows: firstly, the problem and the mathematical models are described including the LBM simulation of hydrodynamic flow, heat transfer and magnetic field. Secondly, the accuracy of the present code is examined in the code validation section. Finally, the results are discussed and a conclusion is given as a closure section.

\section{Mathematical formulation}

Consider the axisymmetric, hydrodynamically fully developed and laminar forced convection flow insider a micro duct filled with porous media. A schematic diagram of the physical model and coordinate system is shown in Fig. 1. In this analysis, the following convectional assumptions are made: flow is incompressible, porous medium is homogenous, isotropic and supposed to be in the Local Thermal Non-Equilibrium condition (LTNE) and all terms of viscous dissipation effects are included. It is also assumed that the fluid is flowing in the slip flow regime, corresponding to Knudsen numbers in the range of $10^{-3} \leq \mathrm{Kn} \leq 10^{-1}$. In the slip flow regime, the velocity slip and temperature jump are applied at the walls of the micro duct. Furthermore, the flow is subjected to a uniform magnetic field. It is assumed that the induced magnetic field produced by the motion of an electrically conducting fluid is negligible compared to the applied magnetic field.

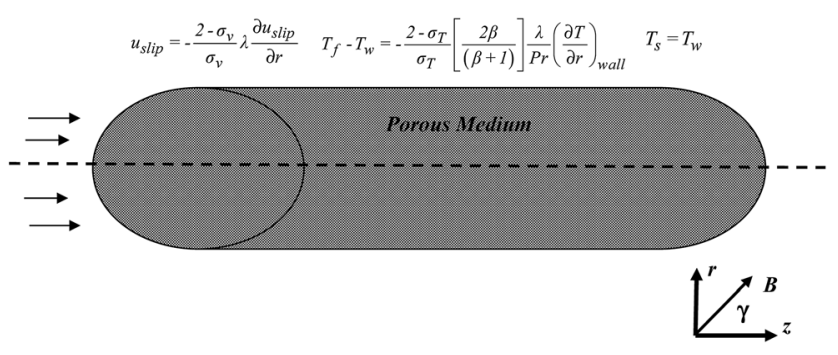

Fig. 1 Geometry and the coordinates system.

\section{Lattice Boltzmann MHD model}

\subsection{Lattice Boltzmann equation for the velocity field}

This section offers a general idea devoted to exploring an extended thermal Lattice Boltzmann model simulating flows under complex conditions. The results of the numerical examples demonstrate the potential of the LBM in dealing with this kind of problems. The LBM has been successfully applied to the simulation of axisymmetric flows. ${ }^{21}$ According the idea of Halliday et al. ${ }^{21}$ in this paper we present a modified Lattice Boltzmann Equation (LBE), in which the source terms were added to the standard 2D LBE. ${ }^{22-26}$

In order to simulate the axisymmetric flows in a simple way, we extend the idea in a consistent manner to the original axisymmetric Lattice Boltzmann model to eliminate the calculations of the velocity gradients. ${ }^{27}$

$$
\begin{aligned}
f_{\alpha}\left(\boldsymbol{z}+e_{\alpha} \Delta t, t+\Delta t\right)-f_{\alpha}(\boldsymbol{z}, t)= & -\tau_{\alpha}\left[f_{\alpha}(\boldsymbol{z}, t)-f_{\alpha}^{\mathrm{eq}}(\boldsymbol{z}, t)\right] \\
& +\omega_{\alpha} \Theta \Delta t+\frac{\Delta t}{\kappa e^{2}} e_{\alpha i} F_{i}
\end{aligned}
$$

In above equations, $f_{\alpha}(z, t)$ is the dynamic distribution function for particles, $\Delta t$ is the time increment, $f_{\alpha}^{\text {eq }}$ is the equilibrium part of distribution function, $f_{\alpha}\left(z+e_{\alpha} \Delta t, t+\Delta t\right)$ is the postcollision distribution function. $z$ is the space vector, i.e., $z=$ $(r, z) ; e=\Delta z / \Delta t ; \Delta z$ is the lattice size; $\omega_{\alpha}$ is the weight given by eqn (7); $\Theta$ is the source or sink term,

$$
\Theta=-\frac{\rho u_{r}}{r}
$$

$F_{i}$ is the force term defined by

$$
F_{i}=-\frac{\rho u_{i} u_{r}}{r}-\frac{2 \rho \nu u_{i}}{r^{2}} \delta_{i r}
$$

$i$ is the index standing for $r$ or $z ; r$ and $z$ are the coordinates in radial and axial directions, respectively; $u_{i}$ is the component of velocity in $i$ direction; $\delta_{i r}$ is the Kronecker $\delta$ function defined by

$$
\delta_{i r}=\left\{\begin{array}{l}
0, i \neq r \\
1, i=r
\end{array}\right.
$$

$e_{\alpha i}$ is the component of $e_{\alpha}$, which represents the velocity vector of a particle in the $\alpha$ link; $\kappa$ is the constant and is determined from

$$
\kappa=\frac{1}{e^{2}} \sum_{\alpha} e_{\alpha z} e_{\alpha z}=\frac{1}{e^{2}} \sum_{\alpha} e_{\alpha r} e_{\alpha r}
$$

And $\tau_{\alpha}$ is an effective relaxation time related to the single relaxation time $\tau^{27}$ as

$$
\tau_{\alpha}=\left\{\begin{array}{l}
\frac{1}{\tau} \quad r=0 \\
\frac{1}{\tau}\left[1+\frac{(2 \tau-1) e_{\alpha r} \Delta t}{2 r}\right] \quad r \neq 0
\end{array}\right.
$$

In this study, the density distribution function $f_{\alpha}^{\mathrm{eq}}$ is modified to consider the magnetic effect. ${ }^{27-30}$ 


$$
\begin{aligned}
f_{\alpha}^{\mathrm{eq}}= & \omega_{\alpha} \rho\left[1+3 \frac{e_{\alpha i} u_{i}}{e^{2}}+\frac{9}{2} \frac{e_{\alpha i} e_{\alpha r} u_{i} u_{r}}{e^{4}}-\frac{3}{2} \frac{u_{i}^{2}}{e^{2}}\right] \\
& +\frac{\omega_{\alpha}}{2 c_{\mathrm{s}}{ }^{2}}\left[\frac{B^{2} e^{2}}{2}-(e B)^{2}\right]
\end{aligned}
$$

where $B$ is the magnetic field and $\omega_{\alpha}$ is the weight coefficient for the D2Q9 model is defined as:

$$
\omega_{\alpha}=\left\{\begin{array}{l}
\frac{4}{9}, \alpha=0 \\
\frac{1}{9}, \alpha=1,3,5,7, \\
\frac{1}{36}, \alpha=2,4,6,8 ;
\end{array}\right.
$$

For axisymmetric D2Q9 model, the nine discrete velocities are defined as following:

$$
e_{\alpha}=\left\{\begin{array}{l}
(0,0), i=0 \\
e(\cos [(\alpha-1) \pi / 4], \sin [(\alpha-1) \pi / 4]), i=1,3,5,7 \\
\sqrt{2} e(\cos [(\alpha-1) \pi / 4], \sin [(\alpha-1) \pi / 4]), i=2,4,6,8
\end{array}\right.
$$

From eqn (4), we have $\kappa=6 .^{27}$

The fluid density $\rho$ and velocity $u_{i}$ are determined from the distribution function in the same manner as that in the Lattice Boltzmann Method for the Navier-Stokes equations:

$$
\rho=\sum_{\alpha} f_{\alpha}, u_{i}=\frac{1}{\rho} \sum_{\alpha} e_{\alpha i} f_{\alpha}
$$

\subsection{Lattice Boltzmann equations for the temperature fields}

Accordingly to Gao et al. ${ }^{31}$ the resolution of the energy equations is written as follows.

$$
\begin{aligned}
g_{\alpha}\left(\boldsymbol{z}+e_{\alpha} \Delta t, t+\Delta t\right)-g_{\alpha}(\boldsymbol{z}, t)= & -\omega_{g}\left[g_{\alpha}(\boldsymbol{z}, t)-g_{\alpha}^{\mathrm{eq}}(\boldsymbol{z}, t)\right]+\Delta t G_{\alpha} \\
& +\Delta t f_{\alpha}(\boldsymbol{z}, t) q_{\alpha}+\Delta t \operatorname{Sr}_{\mathrm{f}, \alpha} \\
h_{\alpha}\left(\boldsymbol{z}+e_{\alpha} \Delta t, t+\Delta t\right)-h_{\alpha}(\boldsymbol{z}, t)= & -\frac{1}{\tau_{h}}\left[h_{\alpha}(\boldsymbol{z}, t)-h_{\alpha}^{\mathrm{eq}}(\boldsymbol{z}, t)\right]+\Delta t \operatorname{Sr}_{s, \alpha}
\end{aligned}
$$

where $g_{\alpha}(z, t)$ and $h_{\alpha}(z, t)$ are respectively the temperature distribution functions for fluid and solid phases. $g_{\alpha}^{\mathrm{eq}}(z, t)$ and $h_{\alpha}(z, t)$ is the equilibrium temperature distribution function and $G_{\alpha}$ is the source term. The relaxation parameter $\omega_{\mathrm{g}}$ is given by ${ }^{32}$

$$
\omega_{\mathrm{g}}=\left[1+\left(e_{i r} \tau_{\mathrm{g}} \Delta t / r\right)\right] /\left(\tau_{\mathrm{g}}+0.5\right)
$$

in which $\tau_{\mathrm{g}}$ is the dimensionless relaxation time related to

$$
\zeta_{\mathrm{e}, \mathrm{f}}=c_{\mathrm{s}}^{2} \tau_{\mathrm{g}} \Delta t
$$

where $\zeta_{\mathrm{e}, \mathrm{f}}$ is designated as the effective thermal diffusivity of the fluid phase.

$\tau_{\mathrm{h}}$ is the relaxation time for the solid phase related to $\zeta_{\mathrm{e}, \mathrm{s}} \mathrm{via}$ the following expression ${ }^{31}$

$$
\zeta_{\mathrm{e}, \mathrm{s}}=\frac{k_{\mathrm{s}, \mathrm{e}}}{(1-\varepsilon)\left(\rho c_{\mathrm{p}}\right)_{\mathrm{s}}}=\Delta t c_{\mathrm{s}}^{2}\left(\tau_{\mathrm{h}}-1 / 2\right)
$$

The single-particle equilibrium temperature distribution function has appropriate form for each phase and expressed $\operatorname{as}^{31,32}$

$$
\begin{gathered}
g_{\alpha}^{\mathrm{eq}}=\omega_{\alpha} T_{\mathrm{f}}\left[1+\frac{e_{\alpha i} u_{i}}{e^{2}}\right] \\
h_{\alpha}^{\mathrm{eq}}=\omega_{\alpha} T_{\mathrm{s}}
\end{gathered}
$$

The source term $G_{\alpha}$ can be chosen as ${ }^{32}$

$$
G_{\alpha}=-\frac{u_{r}}{r} g_{\alpha}^{\mathrm{eq}}
$$

The two discrete heat source terms, $\mathrm{Sr}_{\mathrm{f}, \alpha}$ and $\mathrm{Sr}_{\mathrm{s}, \alpha}$ are given as $^{31}$

$$
\begin{gathered}
\operatorname{Sr}_{\mathrm{f}, \alpha}=\omega_{\alpha}\left[\frac{h_{i}\left(T_{\mathrm{s}}-T_{\mathrm{f}}\right)}{\varepsilon\left(\rho C_{\mathrm{p}}\right)_{\mathrm{f}}}\right] \\
\operatorname{Sr}_{\mathrm{s}, \alpha}=\omega_{\alpha}\left[\frac{h_{i}\left(T_{\mathrm{f}}-T_{\mathrm{s}}\right)}{(1-\varepsilon)\left(\rho C_{\mathrm{p}}\right)_{\mathrm{s}}}\right]
\end{gathered}
$$

According to Shi et al.,$^{33}$ the term associated to viscous heat dissipation is as follows

$$
q_{\alpha}=-\left(f_{\alpha}-f_{\alpha}^{\mathrm{eq}}\right)\left(e_{\alpha}-u\right)\left(e_{\alpha}-u\right): \nabla u
$$

The fluid and solid temperatures are given by:

$$
T_{\mathrm{f}}=\sum_{\alpha} g_{\alpha}, T_{s}=\sum_{\alpha} h_{\alpha}
$$

\subsection{Lattice Boltzmann equations for the magnetic field}

For solving the magnetic field, the following equation must be considered: ${ }^{28-30}$

$$
b_{\alpha}\left(\boldsymbol{z}+e_{\alpha} \Delta t, t+\Delta t\right)-b_{\alpha}(\boldsymbol{z}, t)=-\frac{1}{\tau_{\mathrm{m}}}\left[b_{\alpha}(\boldsymbol{z}, t)-b_{\alpha}^{\mathrm{eq}}(\boldsymbol{z}, t)\right]
$$

where $b_{\alpha}$ represents the magnetic field function and $\tau_{\mathrm{m}}$ is the single relaxation time of the magnetic field and can be defined as: ${ }^{30}$

$$
\eta=c_{\mathrm{s}}^{2}\left(\tau_{\mathrm{m}}-\frac{1}{2}\right)
$$

$\eta$ is the magnetic resistivity.

Similarly, the density equilibrium function $\left(f_{\alpha}^{\mathrm{eq}}\right)$, for calculating the magnetic field, magnetic equilibrium function is considered as follows:

$$
b_{\alpha z}^{\mathrm{eq}}=\omega_{\alpha}\left[B_{z}+\frac{1}{c_{\mathrm{s}}^{2}} e_{\alpha z}\left(u_{r} B_{z}-u_{z} B_{r}\right)\right] \alpha=0, \ldots, 8
$$




$$
b_{\alpha r}^{\mathrm{eq}}=\omega_{\alpha}\left[B_{r}+\frac{1}{c_{\mathrm{s}}^{2}} e_{\alpha r}\left(u_{z} B_{r}-u_{r} B_{z}\right)\right] \alpha=0, \ldots, 8
$$

Finally, microscopic variables are calculated with the following formulas:

$$
B_{z}=\sum_{\alpha} b_{\alpha z} B_{r}=\sum_{\alpha} b_{\alpha r}
$$

\section{Implementation of boundary conditions}

\subsection{Physical boundary conditions}

Boundary conditions are very important for the accuracy and stability of LBM simulation. The unknown particle distribution functions at the boundary nodes must be calculated through proper boundary conditions (Fig. 2).

Two popular kinds of boundary conditions (BC) are used to impose the microscopic conditions on velocity and thermal fields. Velocity slip and the temperature jump are used at wall. $^{15,34}$

$$
\text { Velocity slip : } u_{\text {slip }}=-\frac{2-\sigma_{v}}{\sigma_{v}} \lambda\left(\frac{\partial u_{\text {slip }}}{\partial r}\right)_{\text {wall }}
$$

where $u_{\text {slip }}$ is the velocity slip, $\sigma_{v}$ is the tangential momentum accommodation coefficient and $\lambda$ is the molecular mean free path.

The symmetry condition at $r=0$ :

$$
\frac{\partial u}{\partial r}=0
$$

Boundary conditions of the energy equations include:

The symmetry condition at $r=0$ :

$$
\frac{\partial T_{\mathrm{f}}}{\partial r}=\frac{\partial T_{\mathrm{s}}}{\partial r}=0
$$

At the micro duct wall, the fluid temperature at the wall $T_{\mathrm{f}}$ can be different from that of the wall $T_{\mathrm{w}}$ and this temperature jump can be found as $^{34}$

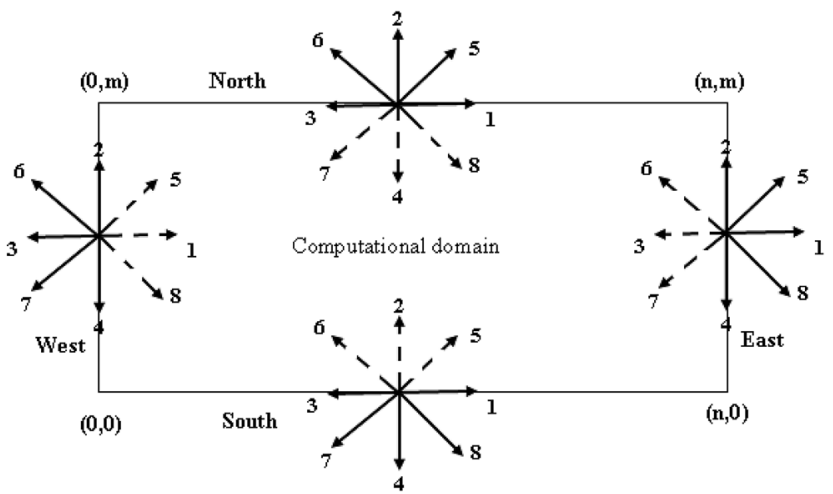

Fig. 2 D2Q9 lattice and velocities.

$$
T_{\mathrm{f}}-T_{\mathrm{w}}=-\frac{2-\sigma_{\mathrm{T}}}{\sigma_{\mathrm{T}}}\left[\frac{2 \beta}{(\beta+1)}\right] \frac{\lambda}{\operatorname{Pr}}\left(\frac{\partial T}{\partial r}\right)_{\text {wall }}
$$

$$
T_{\mathrm{s}}=T_{\mathrm{w}}
$$

where Pr is the Prandtl number, $\beta$ is the specific heat ratio, $\sigma_{\mathrm{T}}$ is the thermal accommodation coefficient.

\subsection{LBM boundary conditions}

4.2.1. Flow. For the north boundary conditions, the slip condition is used ${ }^{35}$ (Fig. 2).

$$
\begin{aligned}
\lambda= & K n^{*} R \\
u_{\text {slip }}= & \lambda \frac{\left(4 u_{\text {slip }, 1}-u_{\text {slip }, 2}\right)}{2+3 \lambda} \\
\rho_{\mathrm{w}}= & f_{1}(\alpha, m)+f_{3}(\alpha, m)+f_{9}(\alpha, m)+2\left(f_{7}(\alpha, m)+f_{4}(\alpha, m)\right. \\
+ & \left.f_{8}(\alpha, m)\right) \\
f_{4}(\alpha, m)= & f_{2}(\alpha, m) \\
f_{7}(\alpha, m)= & \frac{\rho_{\mathrm{w}}\left(1-u_{\text {slip }}\right)-\left(f_{2}(\alpha, m)+f_{4}(\alpha, m)+f_{9}(\alpha, m)\right)}{2} \\
& -\left(f_{3}(\alpha, m)+f_{6}(\alpha, m)\right) \\
f_{8}(\alpha, m)= & \frac{\rho_{\mathrm{w}}\left(1+u_{\text {slip }}\right)-\left(f_{2}(\alpha, m)+f_{4}(\alpha, m)+f_{9}(\alpha, m)\right)}{2} \\
& -\left(f_{1}(\alpha, m)+f_{5}(\alpha, m)\right)
\end{aligned}
$$

For the west boundary conditions, Zou and He condition ${ }^{36}$ is applied if the velocity is known. As for the east, open boundary ${ }^{37}$ is applied.

Another scheme imposed at the axis of the system is the axisymmetric boundary condition of the density distribution function which can be expressed by ${ }^{38}$

$$
f_{2}=f_{4}, f_{5}=f_{8}, f_{6}=f_{7}
$$

4.2.2. Temperature. For the north boundary conditions, the temperature jump conditions is considered ${ }^{35}$

$$
\begin{aligned}
& C_{\mathrm{j}}=\kappa K n=K n \alpha\left(\frac{2 \beta}{(\beta+1) \operatorname{Pr}}\right) \\
& \theta_{\mathrm{f}}=\frac{\left[C_{\mathrm{j}}\left(4 \theta_{R-1}-\theta_{R-2}\right)+2 \theta_{\mathrm{w}}\right]}{2+3 C_{\mathrm{j}}} \\
& g_{4}(\alpha, m)=\theta_{\mathrm{f}}\left(\omega_{2}+\omega_{4}\right)-g_{2}(\alpha, m) \\
& g_{7}(\alpha, m)=\theta_{\mathrm{f}}\left(\omega_{5}+\omega_{7}\right)-g_{5}(\alpha, m) \\
& g_{8}(\alpha, m)=\theta_{\mathrm{f}}\left(\omega_{6}+\omega_{8}\right)-g_{6}(\alpha, m)
\end{aligned}
$$

\section{Entropy generation formulation for thermal non-equilibrium condition}

Due to the existence of thermal non-equilibrium between the solid and fluid phases, the corresponding entropy generation differs from that with the assumption of Local Thermal Equilibrium 
between the two phases. In this section, the entropy generation for thermal non-equilibrium condition is formulated taking account the viscous dissipation effect and the magnetic field. For a point in the porous medium flow, the volumetric rate of the entropy generation for the present study can be expressed as. ${ }^{15,17-19,39,40}$

$$
\begin{aligned}
S_{\mathrm{gen}, \mathrm{s}}^{\prime \prime \prime} & =\frac{k_{\mathrm{s}, e}}{T_{\mathrm{s}}{ }^{2}}\left[\left(\frac{\partial T_{\mathrm{s}}}{\partial z}\right)^{2}+\left(\frac{\partial T_{\mathrm{s}}}{\partial r}\right)^{2}\right]+\frac{h_{i} a_{i}\left(T_{\mathrm{s}}-T_{\mathrm{f}}\right)}{T_{\mathrm{s}}} \\
\dot{S}_{\mathrm{gen}, \mathrm{f}}^{\prime \prime \prime}= & \frac{k_{\mathrm{f}, e}}{T_{\mathrm{f}}{ }^{2}}\left[\left(\frac{\partial T_{\mathrm{f}}}{\partial z}\right)^{2}+\left(\frac{\partial T_{\mathrm{f}}}{\partial r}\right)^{2}\right]+\frac{h_{i} a_{i}\left(T_{\mathrm{s}}-T_{\mathrm{f}}\right)}{T_{\mathrm{f}}} \\
& +\left(\frac{\varepsilon \mu}{K}+\frac{\varepsilon F_{\varepsilon}}{\sqrt{K}}|u|\right) \frac{\left(u^{2}+v^{2}\right)}{T_{\mathrm{f}}} \\
& +\frac{\mu}{T_{\mathrm{f}}}\left\{2\left[\left(\frac{\partial v}{\partial r}\right)^{2}+\left(\frac{v}{r}\right)^{2}+\left(\frac{\partial u}{\partial z}\right)^{2}\right]+\left(\frac{\partial v}{\partial z}+\frac{\partial u}{\partial r}\right)^{2}\right\} \\
& +\frac{\sigma|B|^{2}}{T_{\mathrm{f}}}(u \sin \gamma-v \cos \gamma)^{2}
\end{aligned}
$$

The volumetric rate of entropy generation for thermal nonequilibrium porous medium flow can be obtained by adding eqn (35) and (36) $\dot{S}_{\text {gen }}^{\prime \prime \prime}=\dot{S}_{\text {gen,s }}^{\prime \prime \prime}+\dot{S}_{\text {gen,f }}^{\prime \prime \prime}$ to yield:

$$
\begin{aligned}
\dot{S}_{\mathrm{gen}}^{\prime \prime \prime}= & \frac{k_{\mathrm{s}, e}}{T_{\mathrm{s}}^{2}}\left[\left(\frac{\partial T_{\mathrm{s}}}{\partial z}\right)^{2}+\left(\frac{\partial T_{\mathrm{s}}}{\partial r}\right)^{2}\right]+\frac{k_{\mathrm{f}, e}}{T_{\mathrm{f}}^{2}}\left[\left(\frac{\partial T_{\mathrm{f}}}{\partial z}\right)^{2}+\left(\frac{\partial T_{\mathrm{f}}}{\partial r}\right)^{2}\right] \\
& +\frac{h_{i} a_{i}\left(T_{\mathrm{s}}-T_{\mathrm{f}}\right)^{2}}{T_{\mathrm{s}} T_{\mathrm{f}}}+\left(\frac{\varepsilon \mu}{K}+\frac{\varepsilon F_{\varepsilon}}{\sqrt{K}}|u|\right) \frac{\left(u^{2}+v^{2}\right)}{T_{\mathrm{f}}} \\
& +\frac{\mu}{T_{\mathrm{f}}}\left\{2\left[\left(\frac{\partial v}{\partial r}\right)^{2}+\left(\frac{v}{r}\right)^{2}+\left(\frac{\partial u}{\partial z}\right)^{2}\right]+\left(\frac{\partial v}{\partial z}+\frac{\partial u}{\partial r}\right)^{2}\right\} \\
& +\frac{\sigma|B|^{2}}{T_{\mathrm{f}}}(u \sin \gamma-v \cos \gamma)^{2}
\end{aligned}
$$

The first and second terms of eqn (37) represent heat transfer irreversibility produced by temperature gradients in the solid and the fluid phases, respectively. The third term

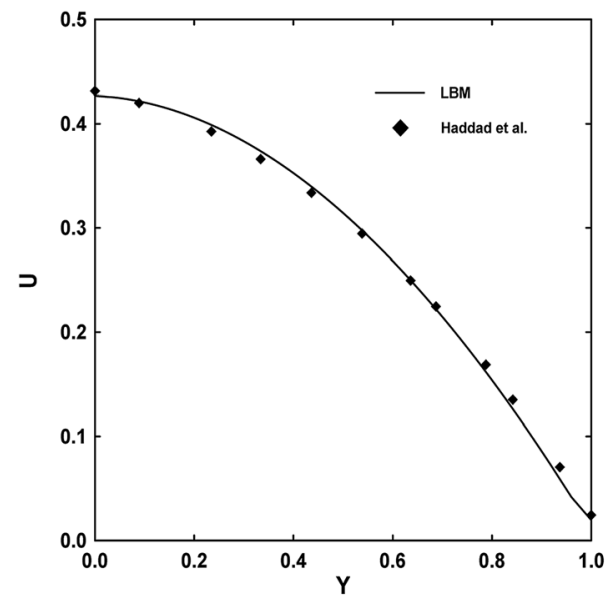

(a)

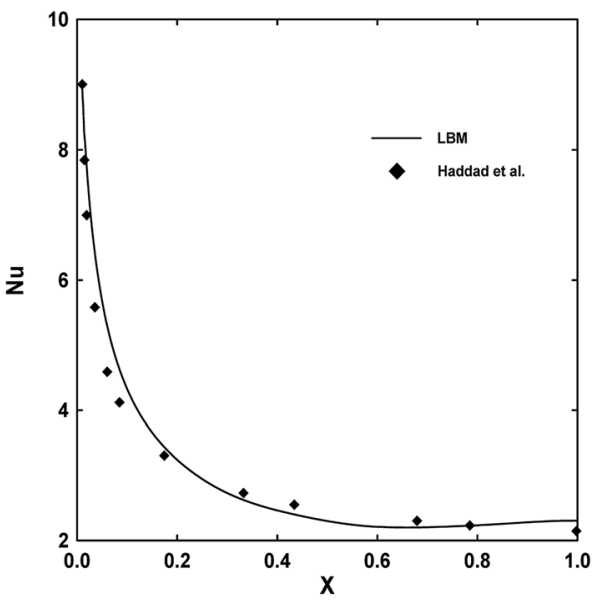

(b)

Fig. 3 Velocity profiles (a) and Nusselt number distributions (b) for case 1 (symbols: Haddad et al. ${ }^{41}$ solid lines: present results (LBM)).

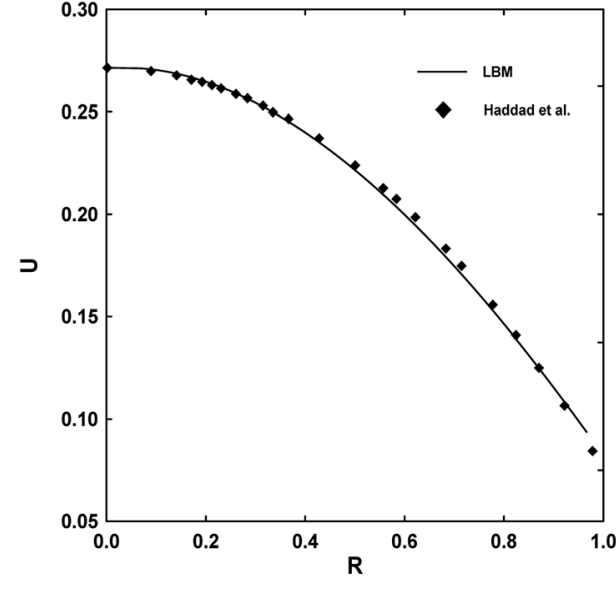

(a)

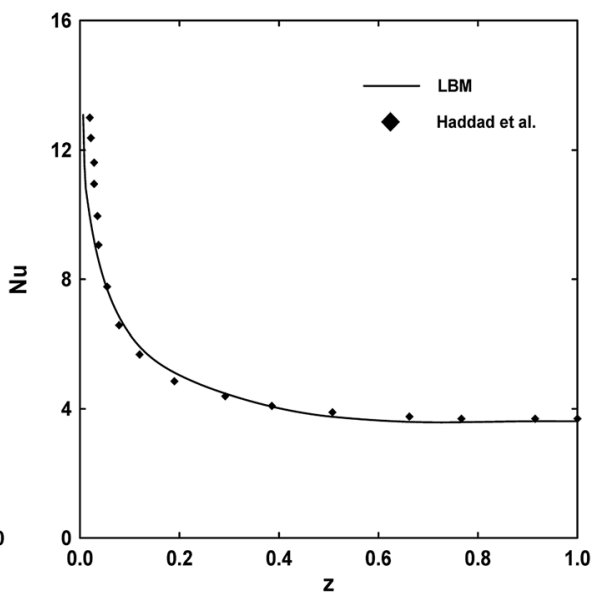

(b)

Fig. 4 Velocity profiles (a) and Nusselt number distributions (b) for case 2 (symbols: Haddad et al., ${ }^{42}$ solid lines: present results (LBM)). 
refers to heat transfer irreversibility generated due to interstitial heat transfer at the solid-fluid interface of the porous medium. The fourth and fifth terms on the equation present the fluid friction irreversibility. The last term is due to the magnetic field. Therefore, the irreversibility components of entropy generation in the porous medium flow can be written as

$$
\begin{aligned}
\dot{S}_{\mathrm{HT}}^{\prime \prime \prime}= & \frac{k_{\mathrm{s}, e}}{T_{\mathrm{s}}{ }^{2}}\left[\left(\frac{\partial T_{\mathrm{s}}}{\partial z}\right)^{2}+\left(\frac{\partial T_{\mathrm{s}}}{\partial r}\right)^{2}\right]+\frac{k_{\mathrm{f}, e}}{T_{\mathrm{f}}{ }^{2}}\left[\left(\frac{\partial T_{\mathrm{f}}}{\partial z}\right)^{2}+\left(\frac{\partial T_{\mathrm{f}}}{\partial r}\right)^{2}\right] \\
& +\frac{h_{i} a_{i}\left(T_{\mathrm{s}}-T_{\mathrm{f}}\right)^{2}}{T_{\mathrm{s}} T_{\mathrm{f}}}
\end{aligned}
$$
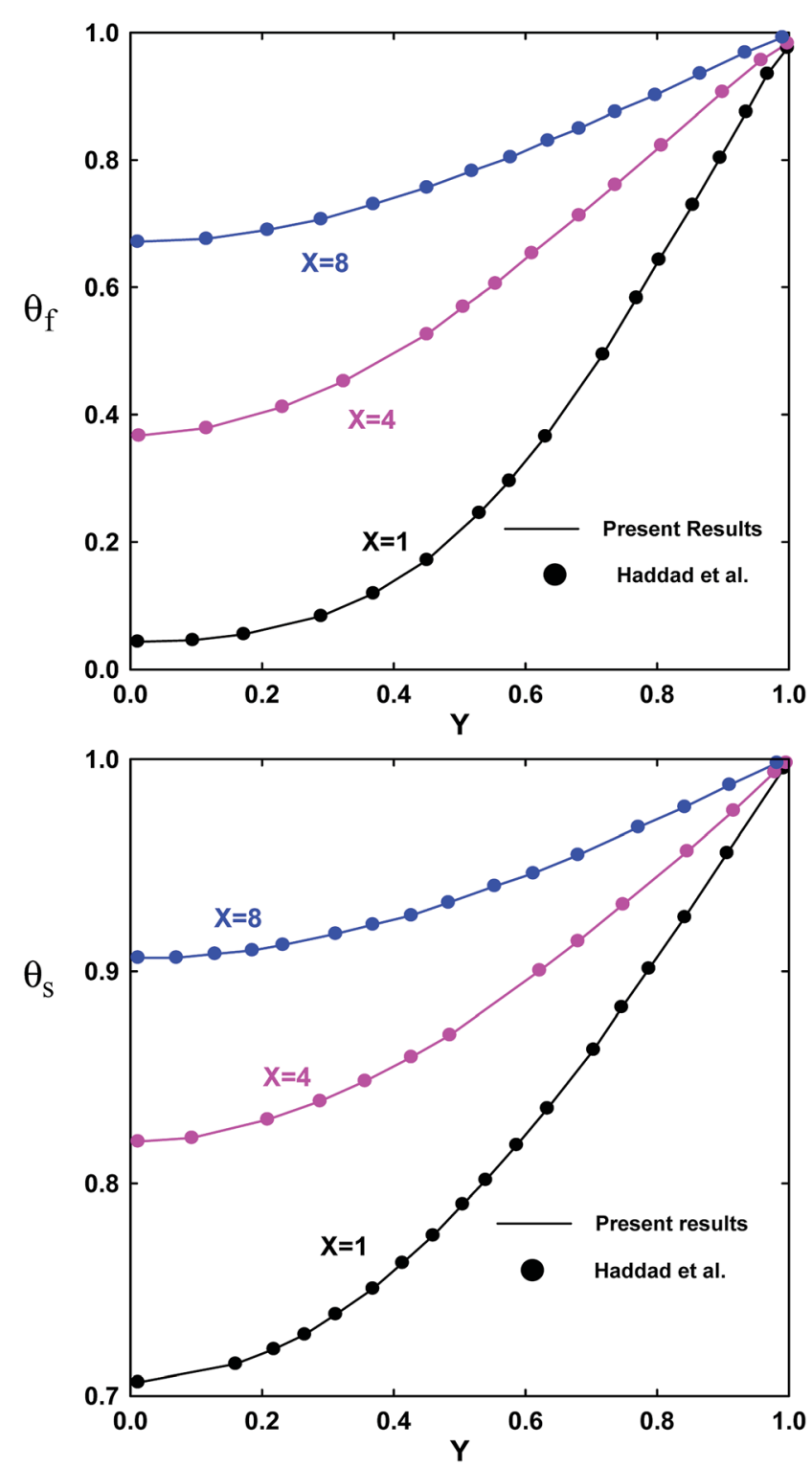

Fig. 5 Fluid and solid temperatures distribution (symbols: Haddad et al., ${ }^{41}$ solid lines: present results (LBM)) for $\mathrm{Kn}=10^{-3}, \mathrm{Da}=1, \mathrm{Bi}=$ 1and $R_{k}=0.1$.

$$
\begin{aligned}
\dot{S}_{\mathrm{FF}}^{\prime \prime \prime}= & \left(\frac{\varepsilon \mu}{K}+\frac{\varepsilon F_{\varepsilon}}{\sqrt{K}}|u|\right) \frac{\left(u^{2}+v^{2}\right)}{T_{\mathrm{f}}} \\
& +\frac{\mu}{T_{\mathrm{f}}}\left\{2\left[\left(\frac{\partial v}{\partial r}\right)^{2}+\left(\frac{v}{r}\right)^{2}+\left(\frac{\partial u}{\partial z}\right)^{2}\right]+\left(\frac{\partial v}{\partial z}+\frac{\partial u}{\partial r}\right)^{2}\right\} \\
& +\frac{\sigma|B|^{2}}{T_{\mathrm{f}}}(u \sin \gamma-v \cos \gamma)^{2}
\end{aligned}
$$

where $\dot{S}_{\mathrm{HT}}^{\prime \prime \prime}$ is the volumetric rate of heat transfer irreversibility and $\dot{S}_{\mathrm{FF}}^{\prime \prime \prime}$ is the volumetric rate of fluid friction irreversibility.

The non-dimensional total entropy generation (Ns) can be expressed as eqn (40)
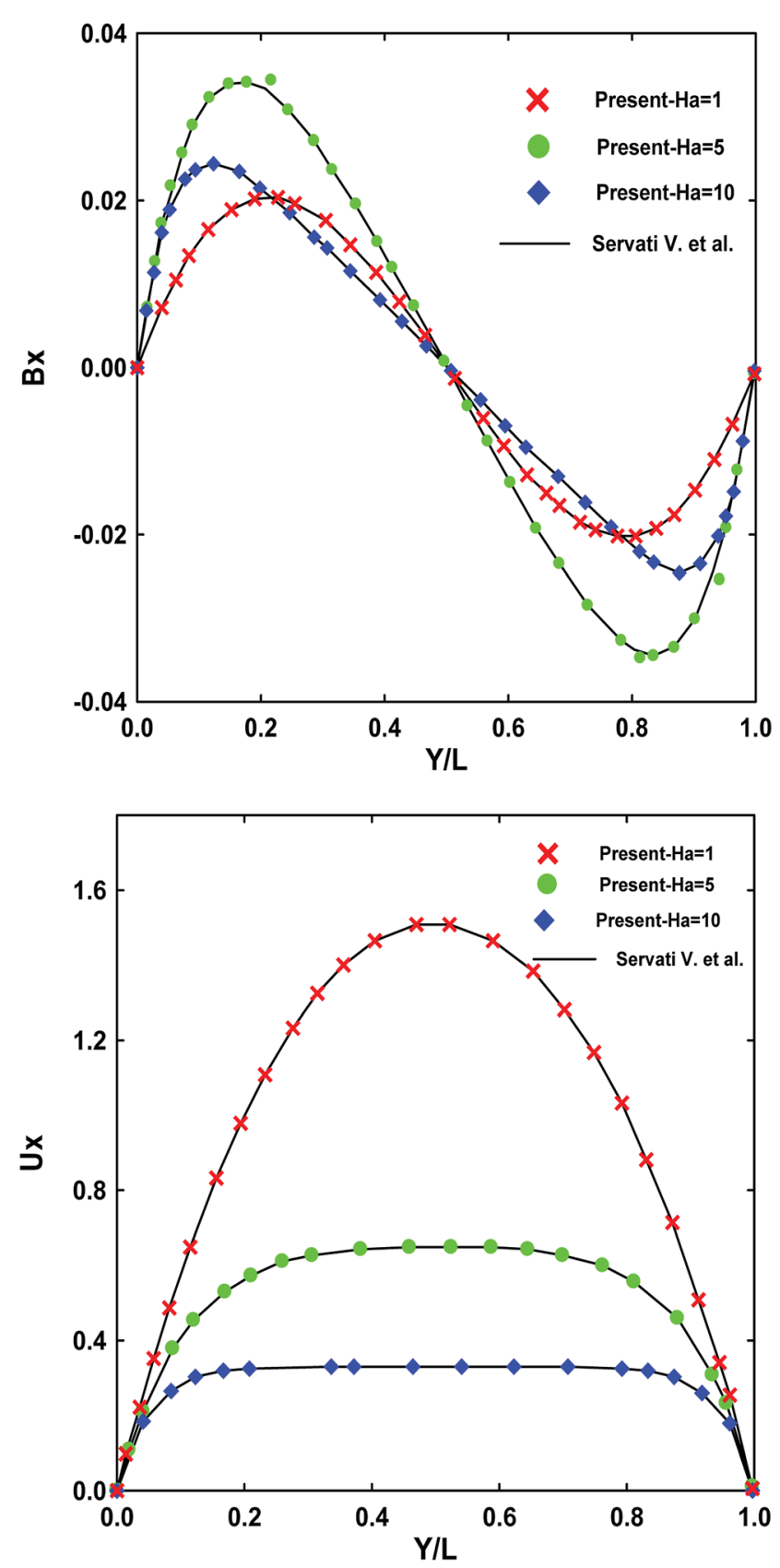

Fig. 6 A comparison of velocity profile (right) and magnetic field (left) in MHD flow between present study and exact analytical solution ${ }^{29}$ for $\mathrm{Ha}=1,5$ and 10. 


$$
\mathrm{Ns}=\frac{\dot{S}_{\mathrm{gen}}^{\prime \prime \prime} R^{2}}{k_{\mathrm{s}, e}}
$$

\section{Code validation}

In order to validate the present numerical code based on the modified LBM, our numerical results were quantitatively compared to the published ones for several cases. The first case concerns a forced convection of gaseous slip-flow in porous micro-channels under Local Thermal Non-Equilibrium conditions carried out by Haddad et al. ${ }^{\mathbf{4 1 , 4 2}}$ The validation is devoted to the velocity and Nusselt number calculation expressed in the following form:

$$
\mathrm{Nu}=\frac{h_{v} L}{\varepsilon k_{\mathrm{f}}}=\frac{1}{1-\theta_{\mathrm{m}}}\left\{\left.\frac{\partial \theta_{\mathrm{f}}}{\partial r}\right|_{\mathrm{w}}+\left.\frac{1}{R_{k}^{*}} \frac{\partial \theta_{s}}{\partial r}\right|_{\mathrm{w}}\right\}
$$

where $\theta_{\mathrm{m}}=\frac{\int_{0}^{1} U \theta r \mathrm{~d} r}{\int_{0}^{1} U r \mathrm{~d} r}$ and $R_{k}^{*}=(1-\varepsilon) k_{\mathrm{s}} / \varepsilon k_{\mathrm{f}}$

Fig. 3 and 4 show the velocity profile and Nusselt number distribution. It is noticeable that the velocity profile is parabolic and that the Nusselt number appears axially downstream in Fig. 4 because less heat transfer occurs from the wall to the fluid. The second case reported on the local thermal non equilibrium of the fluid and solid phases for different Knudsen numbers which is compared with results of Haddad et al. ${ }^{\mathbf{1 1}}$ (Fig. 5).

Another case is also corroborated by the numerical solution ${ }^{29}$ for a fully developed and laminar MHD flow between two parallel plates. In Fig. 6 a good agreement between the present study and the analytical solutions is shown for $\mathrm{Ha}=1,5$ and 10. The results indicate the viability and reliability of the present Lattice Boltzmann model in modeling the MHD flow and fluidsolid coupling heat transfer in complex porous structures.

\section{Results and discussion}

We discussed the effects of different values of magnetic parameter Ha, Knudsen number, Kn, Eckert number, Ec, and Biot number, Bi, on the flow, Nusselt number and entropy generation. The considered slip-flow regime corresponds to Knudsen number ranging from $10^{-3}$ to $10^{-1}$ where the Reynolds number, the Prandtl number and porosity are chosen to be 20, 7 and 0.6 respectively. The default values of the other parameters are mentioned in the description of the respective figures.

\subsection{Velocity distribution}

Fig. 7a and $\mathrm{b}$ describe the effects of Knudsen and Hartmann numbers on the velocity profile. It can be observed from Fig. 7a that an increase in Kn leads to an increase in the velocities slip at walls. Therefore this trend is justified by the fact that an increase in $\mathrm{Kn}$, which is related to a rise in the mean path of the molecule leads to a decrease in the retarding effect at the wall. The effects of Hartmann number $\mathrm{Ha} \mathrm{(1,5}$ and 10) on the dimensionless velocity is shown in Fig. 7b. It can be found that increasing Ha tends to slow down the fluid motion. This trend can be explained by the application of the magnetic field to the micro duct creates a resistance force (Lorentz force) similar to the solid matrix force, which acts in the opposite direction of fluid movement causing the decrease of its speed.

Similarly, Fig. 8 and 9 plot magnetic field for different Knudsen (Kn) and Hartmann (Ha) numbers. It is found that the magnetic field varied with a change in Kn or Ha numbers. It is also observed from this figure that they have the same trend, the classic M-shaped velocity profile commonly observed in micro duct flows. This M-shaped velocity is driven purely by the magnetic field effects, and is the result of the same physical effect as the so-called magnetic collision. In the magnetic collision, the suspended magnetic particles tend to align their dipoles in the direction of the applied field. This particle response may hinder the movement of suspended magnetic particles and hence leads to an increase in the rate of energy
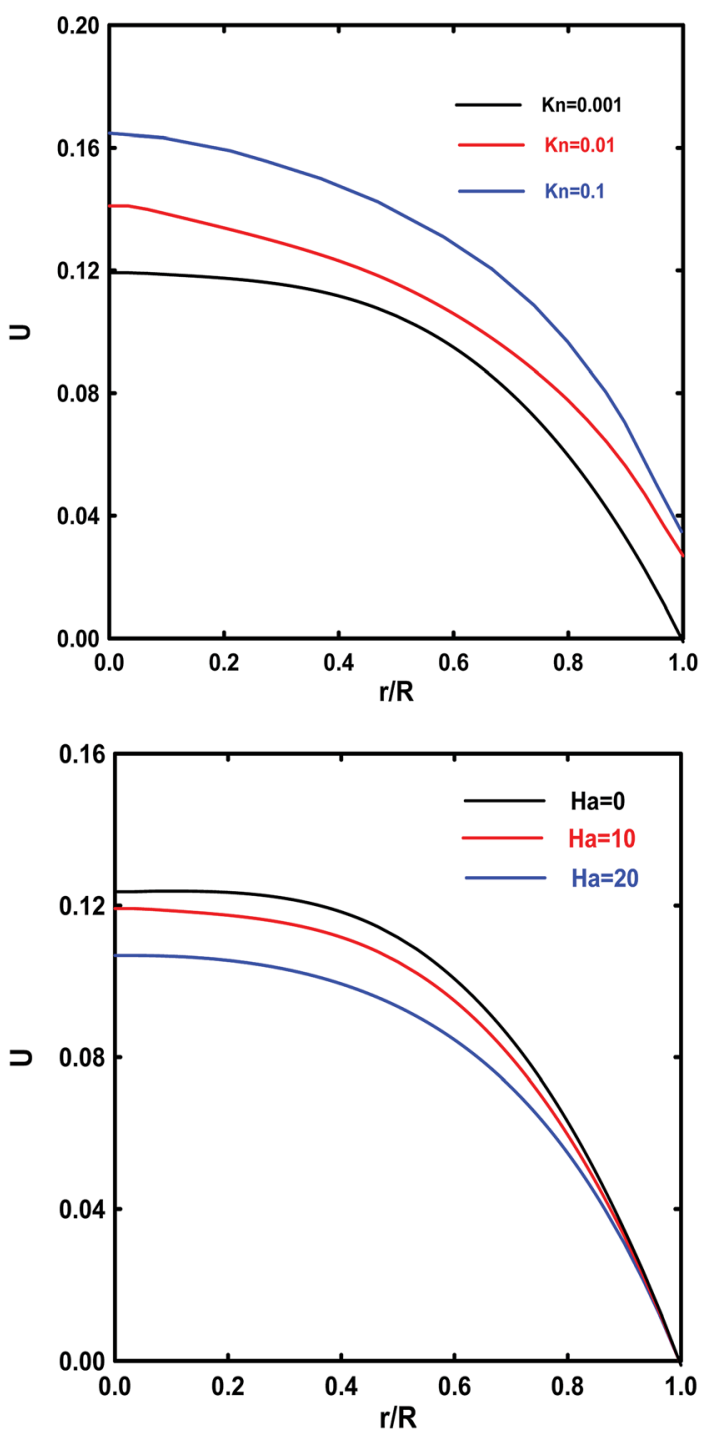

Fig. 7 Effect of $\mathrm{Kn} \mathrm{(a)} \mathrm{and} \mathrm{Ha}$ (b) on the fully developed axial velocity profile. 


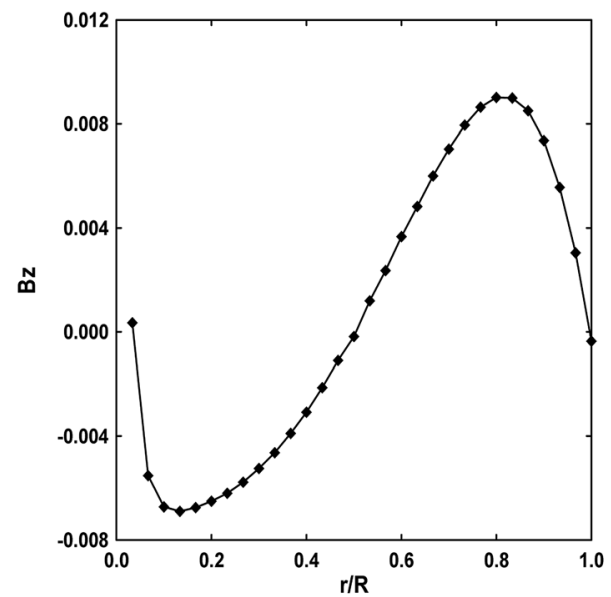

(a)

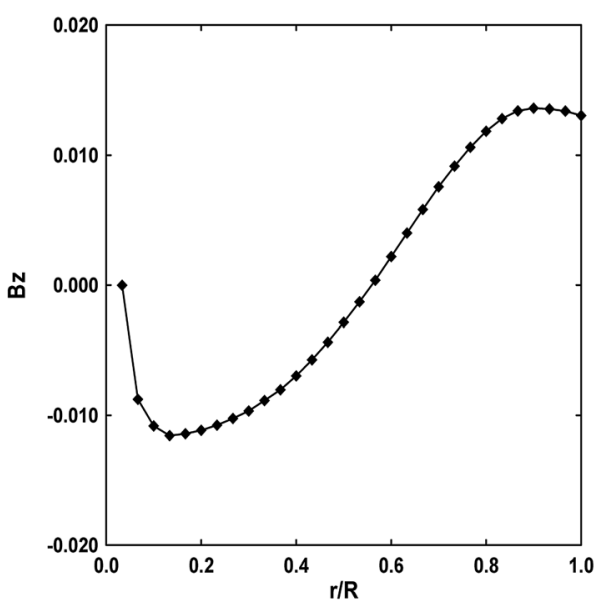

(b)

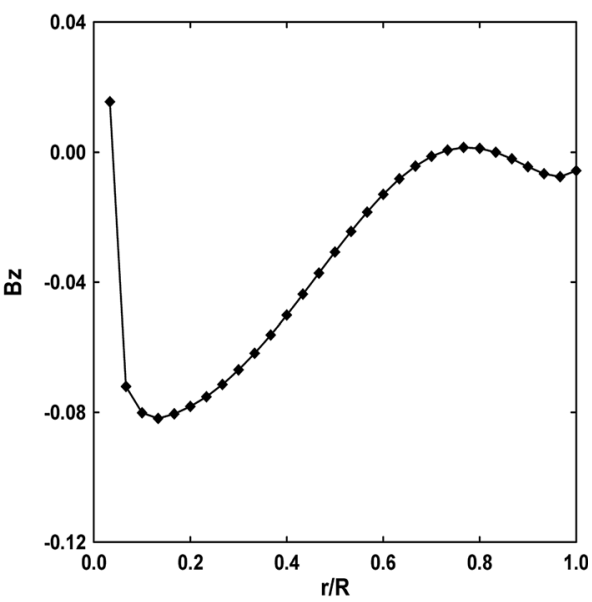

(c)

Fig. 8 Radial variation of the axial magnetic field for (a) $\mathrm{Kn}=10^{-3}$ (b) $\mathrm{Kn}=10^{-2}$ (c) $\mathrm{Kn}=10^{-1}$ for $\mathrm{Ha}=5, \mathrm{Da}=10^{-1}, \mathrm{Ec}=5, \gamma=\pi / 2$ and $R_{k}=5$.

dissipation of the flowing and eventually increases the effective viscosity of the suspension.

\subsection{Nusselt number}

Having derived the velocity, the field and temperature distribution having been derived, we can further determine the Nusselt number $(\mathrm{Nu})$, one of the most important dimensionless parameters in describing the strength of convective heat exchange. The local Nusselt number and the average Nusselt number are defined as follows ${ }^{41,42}$

$$
\mathrm{Nu}=\frac{h_{v} L}{\varepsilon k_{\mathrm{f}}}=\frac{1}{1-\theta_{\mathrm{m}}}\left\{\left.\frac{\partial \theta_{\mathrm{f}}}{\partial r}\right|_{\mathrm{w}}+\left.\frac{1}{R_{k}^{*}} \frac{\partial \theta_{\mathrm{s}}}{\partial r}\right|_{\mathrm{w}}\right\}
$$

where $\theta_{\mathrm{m}}=\frac{\int_{0}^{1} U \theta r \mathrm{~d} r}{\int_{0}^{1} U r \mathrm{~d} r}$ and $R_{k}^{*}=(1-\varepsilon) k_{\mathrm{s}} / \varepsilon k_{\mathrm{f}}$

And the average Nusselt number is:

$$
\operatorname{Nuav}=\frac{1}{S} \iint_{S} \operatorname{Nud} r \mathrm{~d} z
$$

Fig. 10-16 exhibit the influence of the dimensionless parameters ( $\mathrm{Kn}, \mathrm{Ha}, \mathrm{Ec}$ and $\mathrm{Bi}$ ) on local and average Nusselt numbers. It is notable from this figure that the curves have the same tendency. On the other hand, we can see the existence of a critical value of position depending on the emerging parameters $(z<1)$ for which $\mathrm{Nu}$ decreases in a monotonous way. Beyond this position, the Nusselt number tends to be almost constant along the micro-cylinder. The same figure (Fig. 10) shows that as $\mathrm{Kn}$ increases, Nu also increases. This is due to the reduction in heat transfer between the wall and the fluid. A similar behavior is observed for the average Nusselt number (Fig. 11), it improve with the increase of magnetic field. Therefore, the Nusselt number increases from a maximum near the input to an asymptotic value in the developed region of the flow regardless of the Knudsen number. 


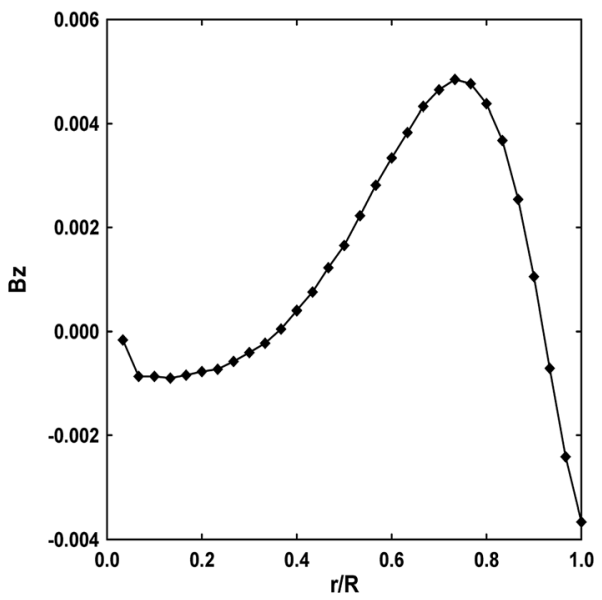

(a)

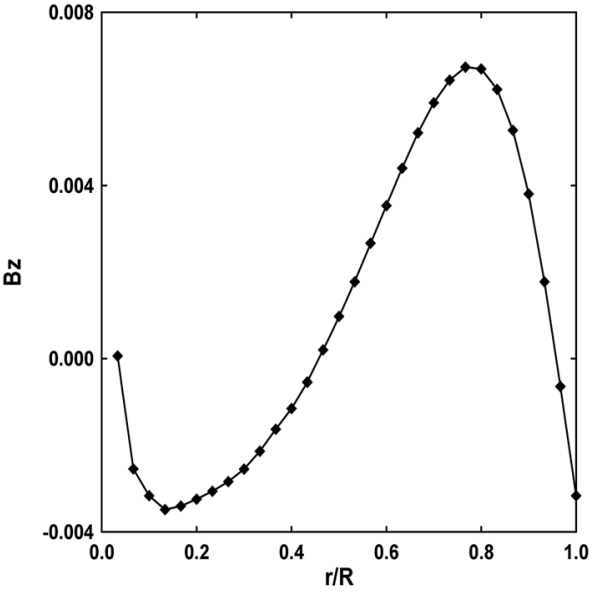

(b)

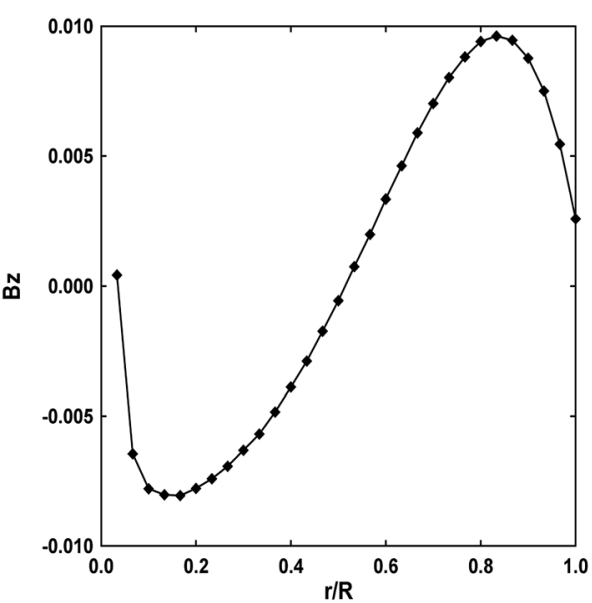

(c)

Fig. 9 Radial variation of the axial magnetic field for (a) $\mathrm{Ha}=1$, (b) $\mathrm{Ha}=5$, (c) $\mathrm{Ha}=10$ for $\mathrm{Kn}=10^{-3}, \mathrm{Da}=10^{-1}, \mathrm{Ec}=5, \gamma=\pi / 2$ and $R_{k}=5$.

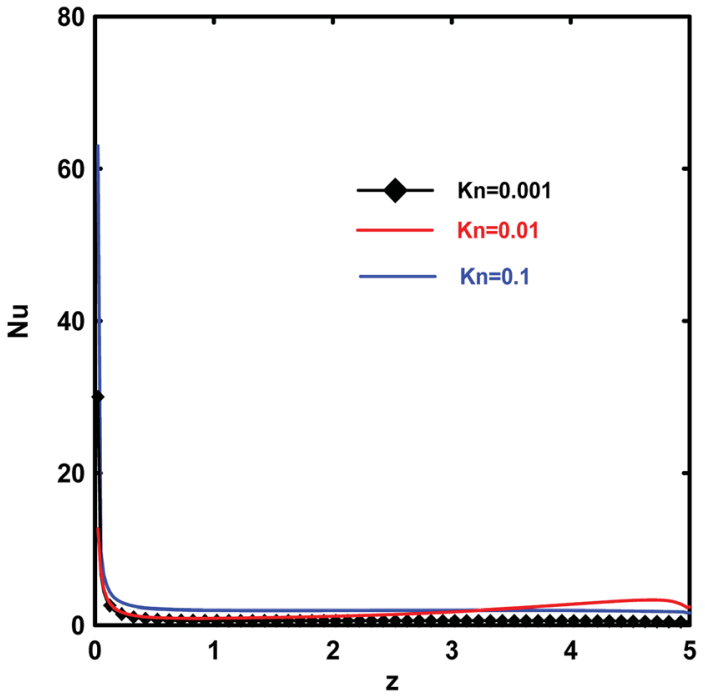

Fig. 10 Effects of $\mathrm{Kn}$ on the local Nusselt number, $\mathrm{Nu}$, for $\mathrm{Kn}=10^{-3}$ $\mathrm{Da}=10^{-1}, \mathrm{EC}=5, \gamma=\pi / 2$ and $R_{k}=5$.

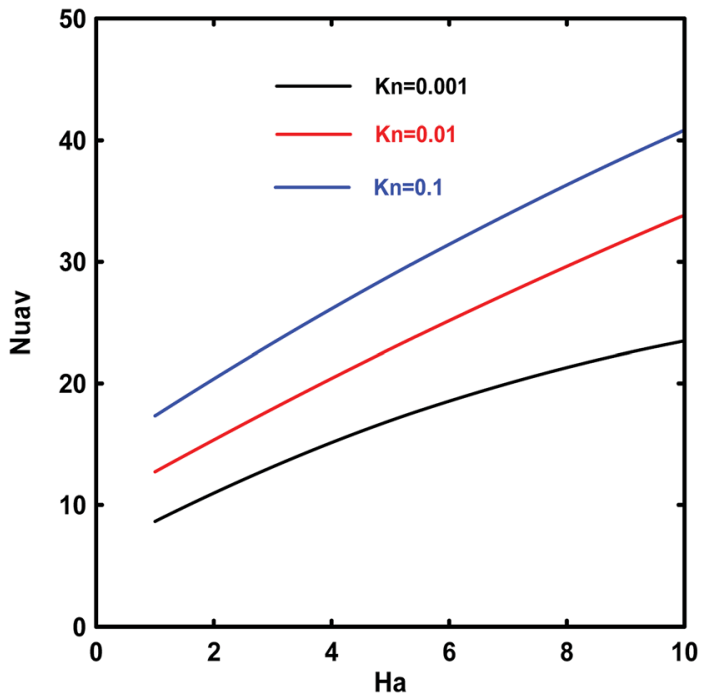

Fig. 11 Effects of Kn on the average Nusselt number, Nuav, vs. Hartmann number $\mathrm{Ha}$ for $\mathrm{Ec}=5, \mathrm{Da}=10^{-1}, \mathrm{Bi}=1, \gamma=\pi / 2$ and $R_{k}=5$. 
Fig. 12 shows the effect of magnetic field on the Nusselt number. For a given fluid and microchannel geometry, changes in Ha mean variations in the magnetic field. Results show that the increase of Hartmann number Ha results in a continuous reduction of Nusselt number $\mathrm{Nu}$. The reason is that increasing Hartmann number causes Lorentz force to increase and leads to a substantial suppression of the convection. Therefore, Nusselt number has a reverse relationship with Hartmann number.

The viscous dissipation effects on the local and average Nusselt numbers through the micro duct are displayed in Fig. 13 and 14. Generally, in a porous medium, viscous dissipation is made up of two components: internal heat generation denoted as Darcy term, and frictional heat generation denoted as Brinkman term. It is observed that the value of Eckert

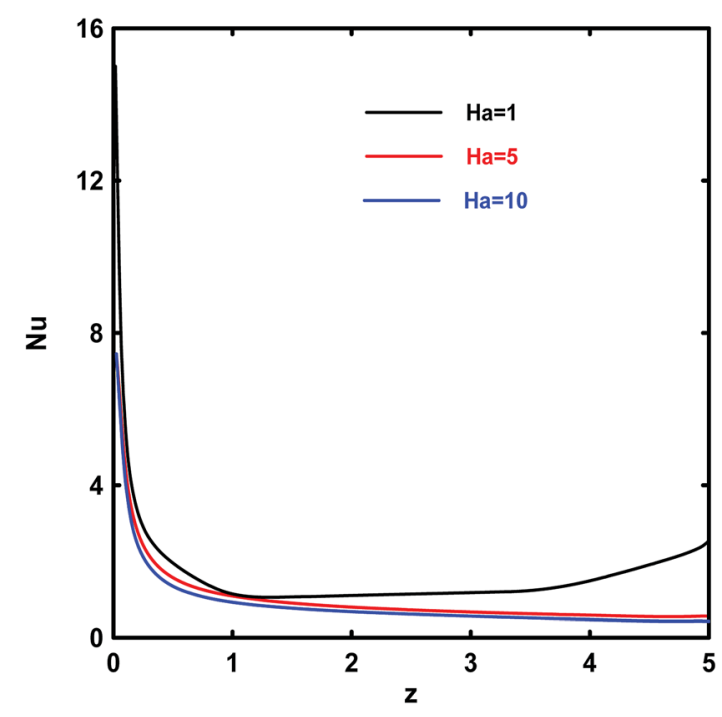

Fig. 12 Effects of $\mathrm{Ha}$ on the local Nusselt number for $\mathrm{Kn}=10^{-3}, \mathrm{Ha}=$ $5, \mathrm{Ec}=5, \gamma=\pi / 2$ and $R_{k}=5$.

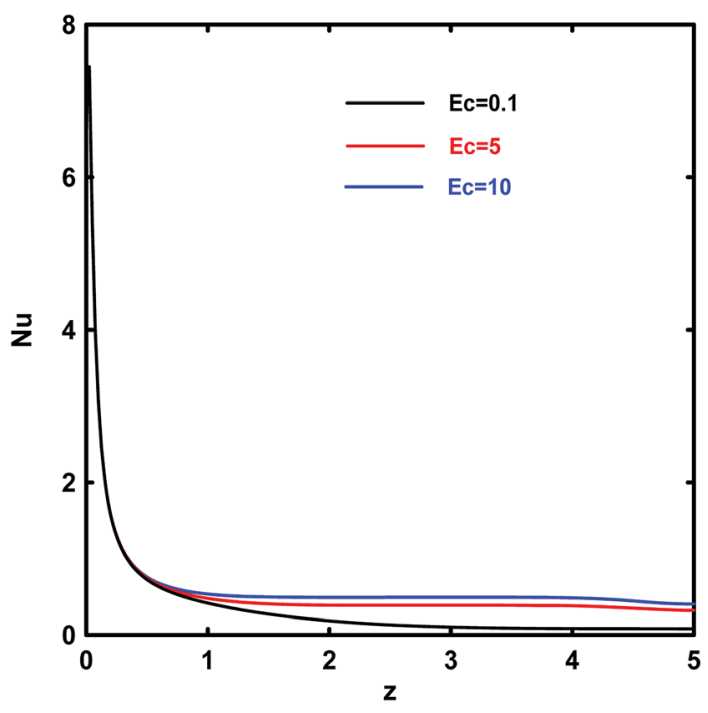

Fig. 13 Effects of Ec on the local Nusselt number for $\mathrm{Kn}=10^{-3}, \mathrm{Da}=$ $10^{-1}, \gamma=\pi / 2$ and $R_{k}=5$. number, Ec, has an accelerating effect on the local $\mathrm{Nu}$. The reason is that the viscous dissipation behaves like an energy source increasing the temperature of the fluid especially near the walls. By increasing the intensity of viscous dissipation, the values of Nuav indicate that the strength of flow and heat transfer flow increases. Therefore, the augmentation of the Eckert number increases the contribution of the heat transfer number due to fluid friction, heat and mass transfer in the micro duct.

The effect of Biot number on the local and average Nusselt number is shown in Fig. 15 and 16. With the increase in Biot number, this number increases gradually. This indicates that the convective heat transfer along the channel walls improves as the convective heat transfer between solid porous matrix and

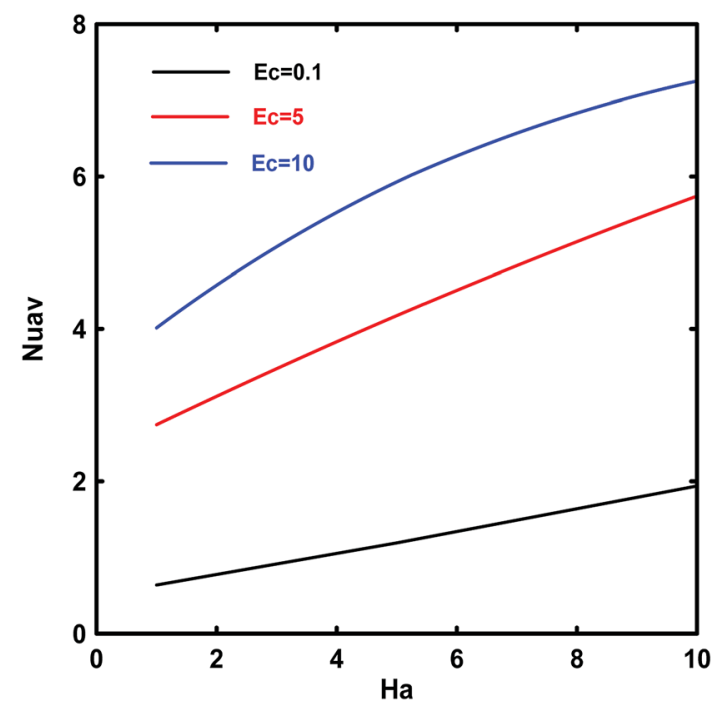

Fig. 14 Effects of Ec on the average Nusselt number, Nuav, vs. Hartmann number $\mathrm{Ha}$ for $\mathrm{Kn}=10^{-3}, \mathrm{Da}=10^{-1}, \mathrm{Bi}=1, \gamma=\pi / 2$ and $R_{k}=5$.

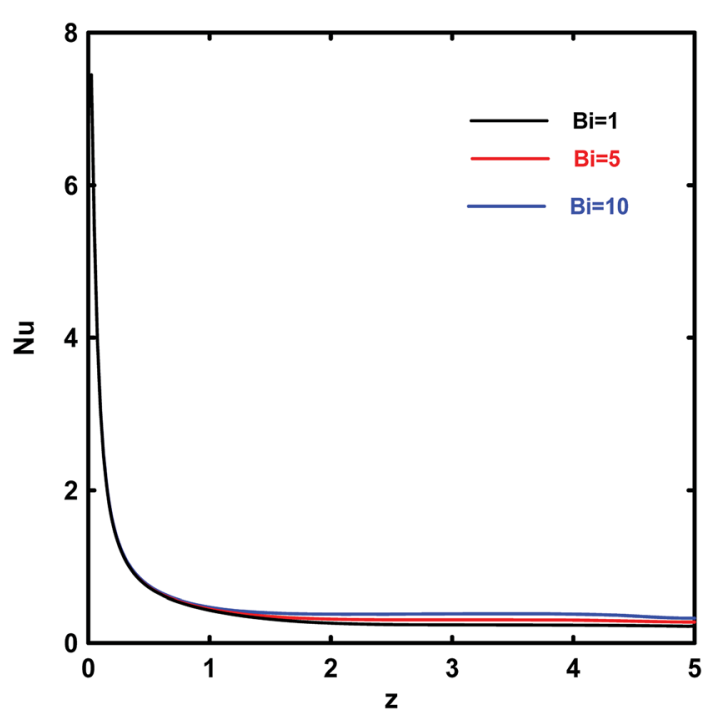

Fig. 15 Effects of Bi on the local Nusselt number for $\mathrm{Da}=10^{-1}, \mathrm{EC}=5$, $\gamma=\pi / 2$ and $R_{k}=5$. 
fluid increases. Moreover, the same behavior appeared on the average Nusselt number.

\subsection{Second-law analysis}

Integrating the volumetric entropy production into the microduct volume, the average entropy generation can be written as:

$$
\mathrm{Nsav}=\frac{1}{s} \iint_{S} N_{s} \mathrm{~d} r \mathrm{~d} z
$$

The local entropy generation, Ns, and average entropy generation, Nsav, are given as a function of different emerging parameters (Fig. 17-23). It is found that all the Ns and Nsav profiles have the same tendency and that two areas are

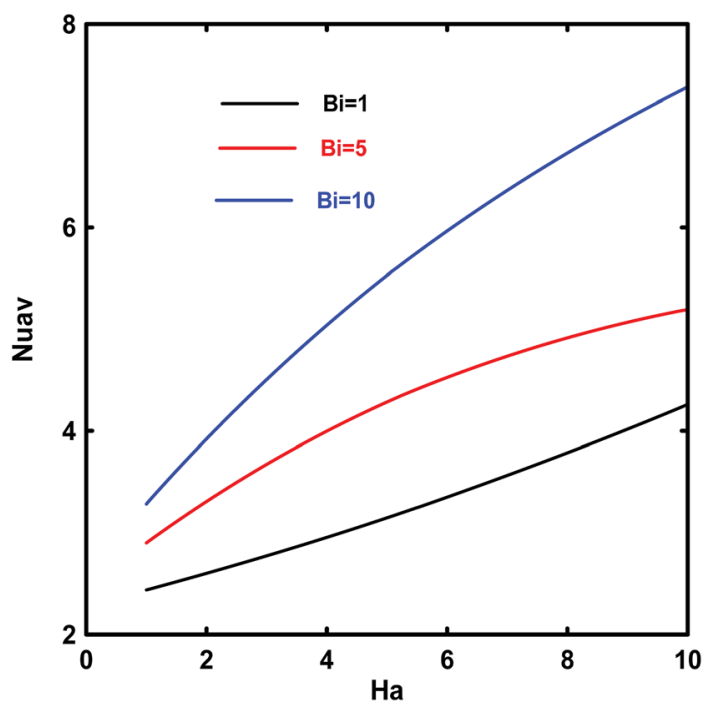

Fig. 16 Effects of $\mathrm{Bi}$ on the average Nusselt number, Nuav, vs. Hartmann number $\mathrm{Ha}$ for $\mathrm{Kn}=10^{-3}, \mathrm{Da}=10^{-1}, \mathrm{EC}=5, \gamma=\pi / 2$ and $R_{k}=5$.

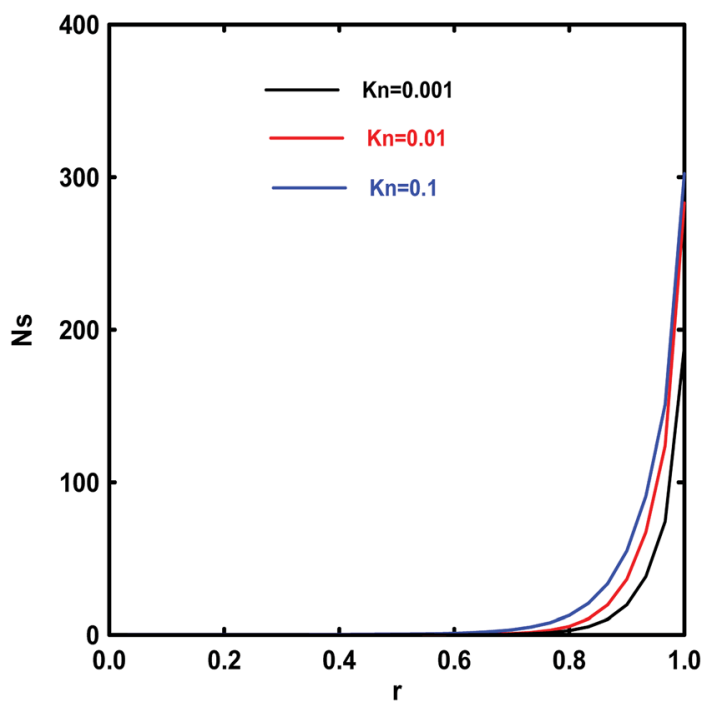

Fig. 17 Effects of Kn on the local entropy generation rate for $\mathrm{Ec}=5$, $\mathrm{Bi}=1, \gamma=\pi / 2$ and $R_{k}=5$. identified: first, a zone with a low gradient of the Ns and Nsav. A second zone with an important gradient. This can be attributed to the increase in temperature gradient as Ha increases. Fig. 17 and 18 illustrate the effect of Kn on the Ns and the Hartmann number on the Nsav as a function of Knudsen number, respectively. As can be seen from these figures that the magnetic field has a great impact on entropy generation. It passes through the duct from a minimum value to a maximum one near the wall. The obtained minimum reached on the axis is due to the smallness of the velocity gradient and because the temperature gradient is nil. This indicates that the FFI dominates the entropy generation rate on the axis. The detected maximum at the wall implies that the velocity slip and temperature jump at the wall have a significant effect on the

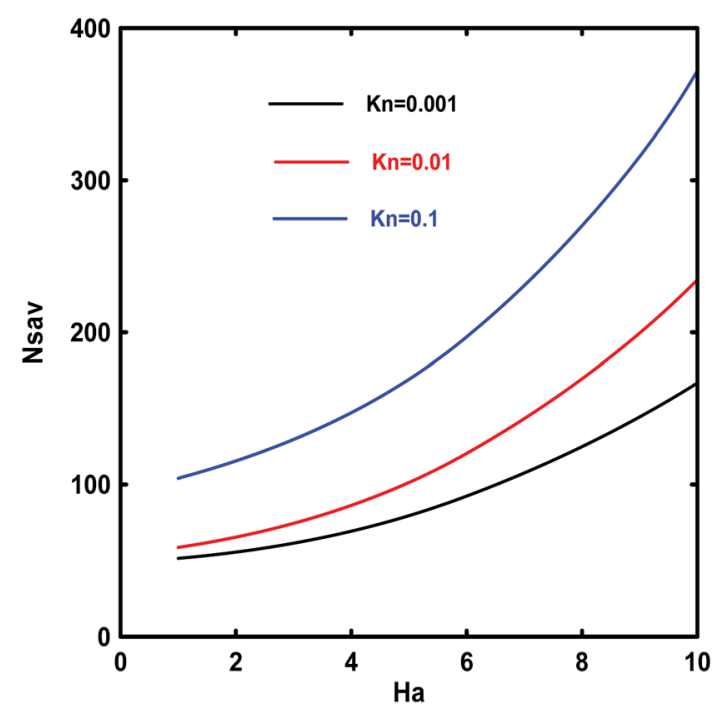

Fig. 18 Effects of $\mathrm{Kn}$ on the average entropy generation, Nsav, vs. Hartmann number $\mathrm{Ha}$ for $\mathrm{EC}=5, \mathrm{Da}=10^{-1}, \mathrm{Bi}=1, \gamma=\pi / 2$ and $R_{k}=5$.

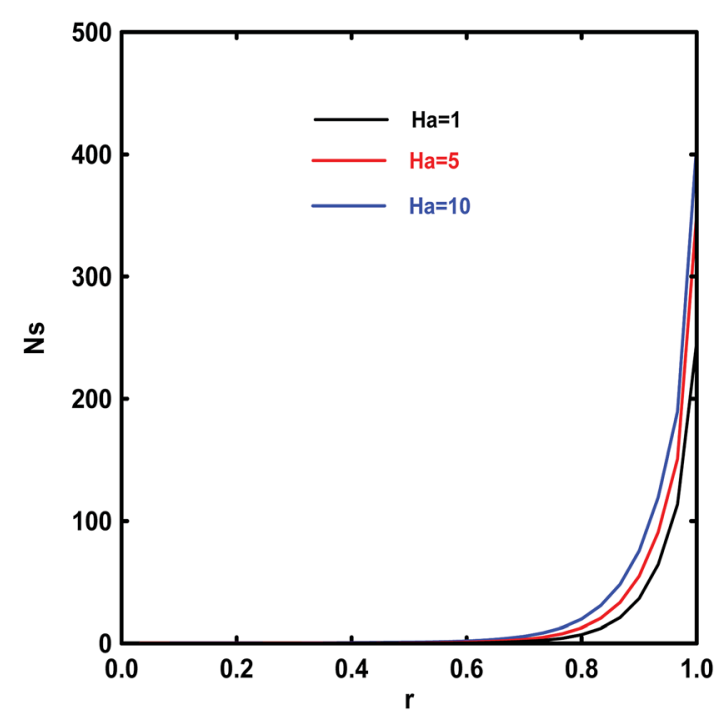

Fig. 19 Effects of $\mathrm{Ha}$ on the local entropy generation rate for $\mathrm{Kn}=$ $10^{-3}, \mathrm{EC}=5, \gamma=\pi / 2$ and $\mathrm{Bi}=1$. 
entropy generation rate. The wall acts as a strong concentrator of irreversibility due to the high value of velocity and temperature gradients occurring at the wall. ${ }^{43}$

The effect of the Hartmann number Ha on the entropy generation number Ns (last term of eqn (44)) are plotted in Fig. 19. An increase of this parameter results in an increase of Ns because the presence of the magnetic field creates more entropy in the fluid. Moreover, the entropy generation number is higher near the wall where both temperature and velocity are at their maximum values. This means that the surface acts as a strong source of irreversibility.

Fig. 20 and 21 show the influence of Ec on Ns and the magnetic field on the average entropy generation Nsav as

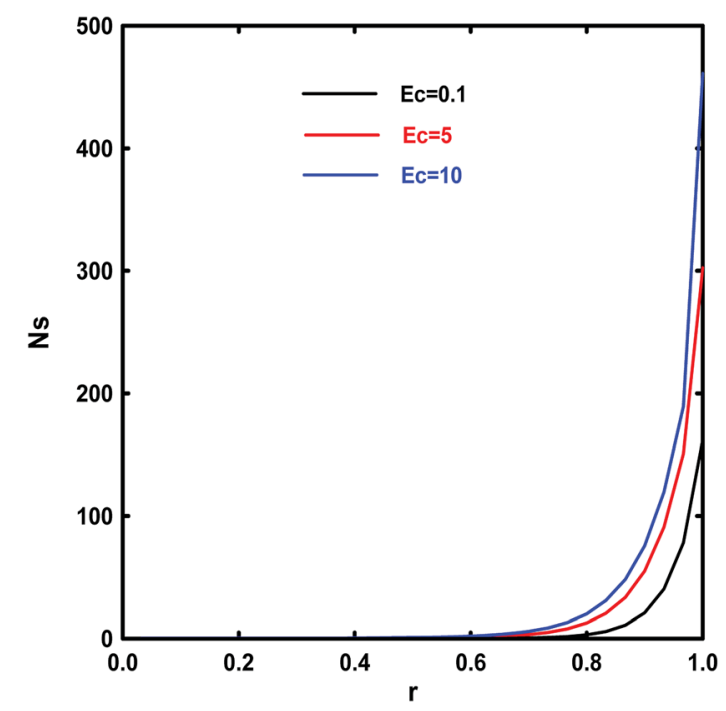

Fig. 20 Effects of Ec on the local entropy generation rate for $\mathrm{Kn}=$ $10^{-3}, \mathrm{Da}=10^{-1}, \gamma=\pi / 2$ and $\mathrm{Bi}=1$.

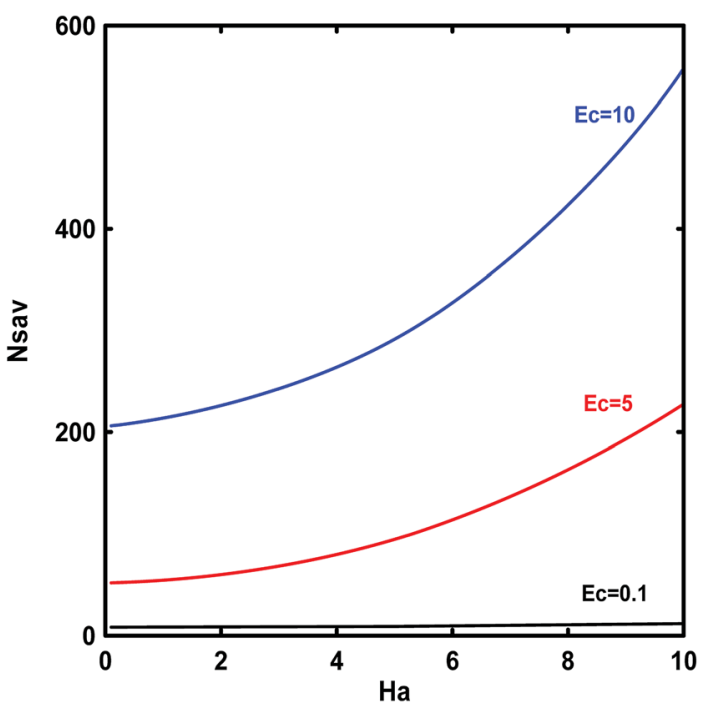

Fig. 21 Effects of Ec on the average entropy generation, Nsav, vs. Hartmann number $\mathrm{Ha}$ for $\mathrm{Kn}=10^{-3}, \mathrm{Da}=10^{-1}, \mathrm{Bi}=1, \gamma=\pi / 2$ and $R_{k}=5$. a function of Ec values, respectively. First of all, it is worthreminding that the entropy generation is involves of friction, heat transfer and magnetic irreversibility's. Consequently, an increase in magnetic field intensity tends to augment the local Ns (Fig. 20). From this figure, it is clear that the Nsav values present a minimum at low Ec values because the entropy generation due to the fluid friction decreases. This indicates that the Ha has no effect when the viscous dissipation is small. For large values of Ha via an increase of magnetic field intensity, the entropy generation grows significantly with Ec. The highest Nsav is observed near the wall; it has a local maximum. One can perceive that the Ha and viscous dissipation effect raise entropy generation along the longitudinal direction of the flow.

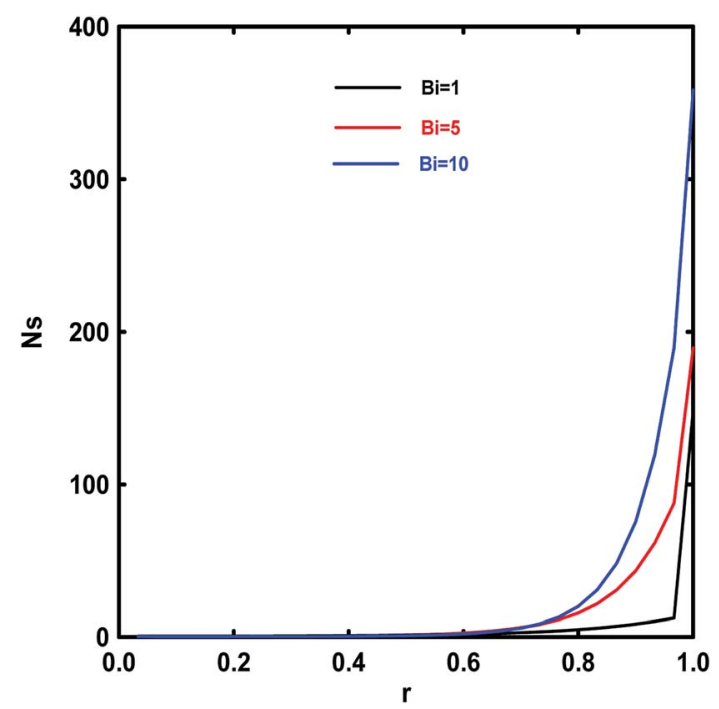

Fig. 22 Effects of $\mathrm{Bi}$ on the local entropy generation rate for $\mathrm{Kn}=$ $10^{-3}, \mathrm{Da}=10^{-1}, \gamma=\pi / 2$.

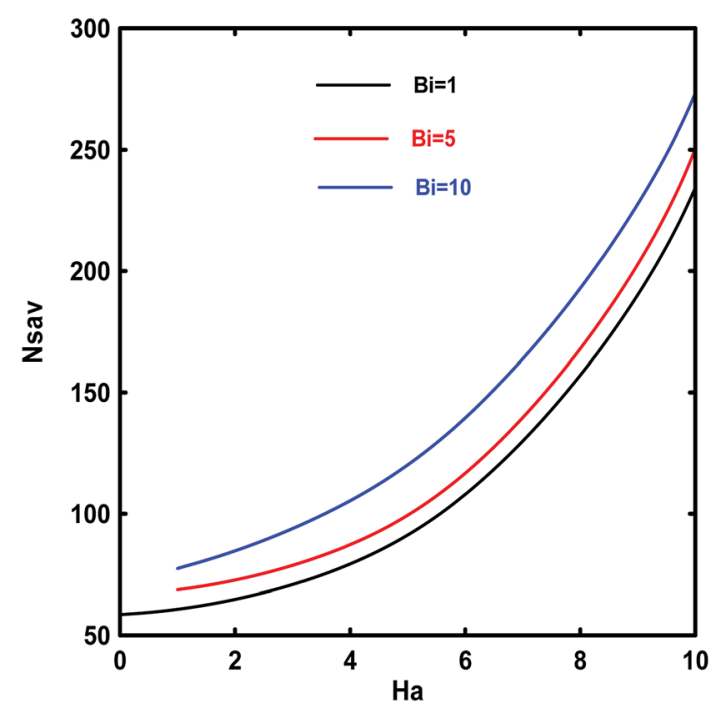

Fig. 23 Effects of $\mathrm{Bi}$ on the average entropy generation, Nsav, vs. Hartmann number $\mathrm{Ha}$ for $\mathrm{Kn}=10^{-3}, \mathrm{Da}=10^{-1}, \mathrm{EC}=5, \gamma=\pi / 2$ and $R_{k}=5$. 
In order to obtain a better understanding of the entropy generation within the LTNE assumption in porous micro duct, we provide the variation of Ns and the effect of increasing magnetic field intensity ( $\mathrm{Ha}$ ) on the local and average entropy generation as a function of Biot number in Fig. 22 and 23. It can be noted that at high $\mathrm{Bi}$, Nsav increases dramatically. The increase in the $\mathrm{Bi}$ number indicates a strong internal heat exchange between the fluid and solid phases causing high irreversibility.

\section{Conclusion}

In this study, fluid flow, magnetic field, Nusselt number and entropy generation in porous micro duct subjected to oriented magnetic field are numerically investigated for a range of Hartmann number, Knudsen number, Eckert number and Biot number. In the current study, the theoretical model is based on LTNE condition including all terms of viscous dissipation effects. The Modified Lattice Boltzmann Method was used to investigate numerically effects of the emerging parameters. To drive the magnetic field, a new development has been introduced for the simulation of MHD flows with the LBM. Using this new technique, we obtained the magnetic field profiles. Moreover, the Nusselt numbers have asymptotic values depending on the emerging parameters for which the convection and conduction heat transfer are equal $(\mathrm{Nu}$ is quasiconstant).

Also, the obtained results of the local and average entropy generation revealed that for all parameters $(\mathrm{Kn}, \mathrm{Ha}, \mathrm{Ec}, \mathrm{Bi})$ the average entropy generation is intensified near the wall of the duct via the highest of temperature and velocity gradients in this region. This is confirmed that the wall represents a concentrator of irreversibility. It is also found that the presence of an external oriented magnetic field has strongly affected the global irreversibility. There is a critical value of Ha for which the global irreversibility is quasi constant depending on the emerging parameters.

\section{Nomenclature}

$\begin{array}{ll}a_{i} & \text { Specific surface area }\left(\mathrm{m}^{-1}\right) \\ B & \text { Magnetic field }(\mathrm{T}) \\ \mathrm{Bi} & \text { Biot number, Bi }=a_{i} h_{i} R^{2} / k_{\mathrm{s}} \\ C_{\mathrm{p}} & \text { Specific heat capacity at constant pressure, }\left(\mathrm{J} \mathrm{kg}^{-1} \mathrm{~K}^{-1}\right) \\ c_{\mathrm{S}} & \text { Sound speed, }\left(\mathrm{m} \mathrm{s}^{-1}\right) \\ \mathrm{Da} & \text { Darcy number, Da }=K / R^{2} \\ \mathrm{Ec} & \text { Eckert number, Ec }=u_{\mathrm{o}}{ }^{2} / C_{\mathrm{f}} \Delta T_{\mathrm{ref}} \\ e & \text { Lattice speed, }\left(\mathrm{m} \mathrm{s}{ }^{-1}\right) \\ e_{\alpha} & \text { Discrete velocity, }\left(\mathrm{m} \mathrm{s}{ }^{-1}\right) \\ F_{\varepsilon} & \text { Forchheimer form coefficient } \\ f_{\alpha} & \text { Density distribution function } \\ f_{\alpha}^{\text {eq }} & \text { Equilibrium distribution function of density } \\ F_{i} & \text { Force terme } \\ g_{\alpha} & \text { Fluid temperature distribution function } \\ g_{\alpha}^{\text {eq }} & \text { Equilibrium fluid temperature distribution function } \\ \text { Ha } & \text { Hartmann number, Ha }=B_{o} R / \sqrt{\nu \eta}\end{array}$

\section{Greek symbols}

$\begin{array}{ll}\Delta z & \text { Lattice step, }(\mathrm{m}) \\ \Delta t & \text { Time step, }(\mathrm{s}) \\ \gamma & \text { Magnetic field inclination } \\ \nu & \text { Kinematic viscosity } \\ \sigma & \text { Electrical conductivity } \\ \zeta & \text { Thermal diffusivity, }\left(\mathrm{m}^{2} \mathrm{~s}^{-1}\right) \\ \varepsilon & \text { Porosity of porous media } \\ \mu & \text { Dynamic fluid viscosity, }\left(\mathrm{kg} \mathrm{m}^{-1} \mathrm{~s}^{-1}\right) \\ \lambda & \text { Molecular mean free path, }\left(\mathrm{m}^{-1}\right. \\ \rho & \text { Density, }\left(\mathrm{kg} \mathrm{m}^{-3}\right) \\ \sigma_{v} & \text { Tangential momentum accommodation coefficient } \\ \sigma_{T} & \text { Thermal accommodation coefficient } \\ \tau & \text { Dimensionless relaxation time } \\ \theta_{\mathrm{m}} & \text { Mixing cup temperature } \\ \omega_{\alpha} & \text { Weight coefficient } \\ \beta & \text { Specific heat transfer ratio } \\ \eta & \text { Magnetic resistivity }\end{array}$

\section{Subscripts}

$\begin{array}{ll}\text { e } & \text { Effective or equivalent } \\ \text { f } & \text { Fluid } \\ \text { S } & \text { Solid. }\end{array}$

\section{References}

1 B. Buonomo, G. Lauriat, O. Manca and S. Nardini, Int. J. Heat Mass Transfer, 2016, 98, 484.

2 M. Dehghan, M. S. Valipour and S. Saedodin, Energy Convers. Manage., 2015, 91, 259.

3 S. Qian and H. H. Bau, Mech. Res. Commun., 2009, 36, 10.

4 J. Jang and S. S. Lee, Sens. Actuators, A, 2000, 80, 8489.

5 Y. Jian, Int. J. Heat Mass Transfer, 2015, 89, 193.

6 C. Das, G. Wang and F. Payne, Sens. Actuators, A, 2013, 201, 43. 
7 R. Ellahi, Appl. Math. Model., 2013, 37, 1451.

8 S. M. Aminossadati, A. Raisi and B. Ghasemi, International Journal of Non-Linear Mechanics, 2011, 46, 1373.

9 V. Sofonea and W. G. Frueh, Eur. Phys. J. B, 2001, 20, 141.

10 Y. Xuan, M. Ye and Q. Li, Int. J. Heat Mass Transfer, 2005, 48, 2443.

11 P. J. Dellar, J. Comput. Phys., 2002, 179, 95.

12 P. J. Dellar, J. Stat. Mech.: Theory Exp., 2009, 2009, P06003.

13 C. X. Wang and S. B. Chang, Chin. Phys., 2005, 14, 1398.

14 M. J. Pattison, K. N. Premnath, N. B. Morley and M. A. Abdou, Fusion Eng. Des., 2008, 83, 557.

15 T. W. Ting, Y. M. Hung and N. Guo, Int. J. Heat Mass Transfer, $2015,81,862$.

16 A. C. Baytas, J. Porous Media, 2007, 10, 261.

17 B. Buonomo, O. Manca and G. Lauriat, Int. J. Therm. Sci., 2014, 77, 206.

18 M. Torabi, K. Zhang, G. Yang, J. Wang and P. Wu, Energy, 2015, 82, 922.

19 T. W. Ting, Y. M. Hung and N. Guo, Energy Convers. Manage., 2015, 105, 731.

20 L. J. Betchen and A. G. Straatman, Numer. Heat Transfer, Part $B, 2008,53,412$.

21 I. Halliday, L. A. Hammond, C. M. Care, K. Good and A. Stevens, Phys. Rev. E: Stat., Nonlinear, Soft Matter Phys., 2001, 64, 011208.

22 T. Reis and T. N. Phillips, Phys. Rev. E: Stat., Nonlinear, Soft Matter Phys., 2008, 77, 026703.

23 Q. Li, Y. L. He, G. H. Tang and W. Q. Tao, Phys. Rev. E: Stat., Nonlinear, Soft Matter Phys., 2009, 80, 037702.

24 H. Huang and X. Y. Lu, Phys. Rev. E: Stat., Nonlinear, Soft Matter Phys., 2009, 80, 016701.

25 T. Reis and T. N. Phillips, Phys. Rev. E: Stat., Nonlinear, Soft Matter Phys., 2007, 75, 056703.

26 X. D. Niu, C. Shu and Y. T. Chew, Int. J. Mod. Phys. C, 2003, 14, 785 .
27 J. G. Zhou, Phys. Rev. E: Stat., Nonlinear, Soft Matter Phys., 2011, 84, 036704.

28 H. R. Ashorynejad, A. A. Mohamad and M. Sheikholeslami, Int. J. Therm. Sci., 2013, 64, 240.

29 A. A. V. Servati, K. Javaherdeh and H. R. Ashorynejad, Adv. Powder Technol., 2014, 25, 666.

30 A. K. Husseina, H. R. Ashorynejadb, M. Shikholeslamib and S. Sivasankaran, Nucl. Eng. Des., 2014, 268, 10.

31 D. Gao, Z. Chen and L. Chen, Int. J. Heat Mass Transfer, 2014, 70, 979.

32 Q. Liu, Y. L. He and Q. Li, J. Comput. Phys., 2014, arXiv:1409.5929.

33 Y. Shi, T. S. Zhao and Z. L. Guo, Phys. Rev. E: Stat., Nonlinear, Soft Matter Phys., 2004, 70, 066310.

34 Y. Mahmoudi, Int. J. Heat Mass Transfer, 2015, 85, 524.

35 S. B. Celik, A thesis submitted to the graduate school of natural and applied sciences of Middle East technical university, 2012. 36 Q. Zou and X. He, Phys. Fluids, 1997, 6, 1591.

37 A. Mohamad, Lattice Boltzmann Method Fundamentals And Engineering Applications with Computer Codes, Schulich School of Engineering, the University of Calgary, Alberta, Canada, 2011, pp. 2-31, ISBN 978-0-9783253-0-5.

38 F. Rong, Z. Guo, Z. Chai and B. Shi, Int. J. Heat Mass Transfer, 2010, 53, 5519.

39 F. Selimefendigil, H. F. Öztop and A. J. Chamkha, J. Magn. Magn. Mater., 2016, 406, 266.

$40 \mathrm{H}$. Dhahri, K. Slimi and S. Ben Nasrallah, Comput. Therm. Sci., 2010, 2, 381.

41 O. M. Haddad, M. A. Al-Nimr and J. Sh. Al-Omary, Transp. Porous Media, 2007, 67, 453.

42 O. M. Haddad, M. A. Al-Nimr and M. S. Sari, Forced convection gaseous slip flow in circular porous microchannels, Transp. Porous Media, 2007, 70, 167.

43 H. Dhahri, A. Boughamoura and S. BenNasrallah, J. Porous Media, 2013, 16, 69. 